\title{
Annual Normalized Difference Vegetation Index time-series data for Australian statistical areas
}

Authors

Simon Ramsey ${ }^{1}$, Suzanne Mavoa ${ }^{1}$

Affiliations

1. Melbourne School of Population \& Global Health, University of Melbourne, Australia

corresponding author: Suzanne Mavoa (suzanne.mavoa@unimelb.edu.au)

\begin{abstract}
Google Earth Engine provides researchers with a platform for conducting planetary scale analysis of environmental processes and landcover change, both by providing the necessary tools and by handling the large quantities of data these analyses require. The most widely used moderate-resolution sensors, onboard the Landsat satellite platforms, often require preprocessing to prepare the data for analysis. This data set consists of Australia-wide Landsat derived Normalised Difference Vegetation Index (NDVI) values for the years 2001-2019. The median annual NDVI value for each Statistical Area 1 (SA1) and Statistical Area 2 (SA2) were calculated, and statistics for this imagery is provided in a tabular format. The accompanying Google Earth Engine script applies the pre-processing steps required to account for sensor, solar and atmospheric effects, improving continuity between imagery across space and time and therefore, will enable researchers beyond the remote sensing community to access analysis-ready imagery for the moderate resolution Landsat and Sentinel-2 satellite platforms.
\end{abstract}

\section{Background \& Summary}

Earth observation data is an invaluable resource for environmental monitoring and change detection. As such it has the potential to meet the increasing demand from diverse disciplines for high spatiotemporal resolution data to measure and monitor landcover change. With the advent of Google Earth Engine ${ }^{1}$, the high computational costs associated with processing satellite data are largely removed. However, knowledge gaps remain a barrier to the appropriate and robust use of earth observation data.

Since the launch of the first Landsat satellite in 1972 by the National Aeronautics and Space Administration (NASA), and the successful launch of six subsequent Landsat satellites, a near continuous record of satellite imagery has been available for global environmental monitoring. The Landsat program provides the longest temporal record of space-based surface observations of reflective and thermal wavelength imagery with increasing spectral and spatial fidelity with each new generation of sensor ${ }^{2}$. The entire Landsat data archive, including all future acquisitions, became freely available in early 2009 , resulting in a 50 -fold annual increase in image downloads ${ }^{3}$.

\section{Atmospheric correction}

The accuracy of satellite imagery is impacted by the Earth's atmosphere. As radiation emitted by the sun travels through the Earth's atmosphere, it is scattered and absorbed by atmospheric gases and aerosols, which impacts on the radiation spectrum before it reaches the sensor. Scattering is highest in the blue wavelengths and decreases exponentially towards the near infrared. To account for this, algorithms such as the Landsat Ecosystem Disturbance Adaptive Processing System (LEDAPS) ${ }^{4}$ and the Land Surface Reflectance Code (LaSRC) ${ }^{5}$ have been developed to atmospherically correct Landsat imagery to produce a product known as surface reflectance. Surface reflectance corrects for atmospheric effects such as aerosol scattering or cirrus clouds to improve comparison between successive scenes. The initial signal 
recorded at the sensor are calibrated to radiance values, a measure of the energy flux recorded at the sensor, then rescaled to digital numbers (DNs) in either 8-bit, for the ETM and ETM+ sensors onboard Landsat 5 and 7, respectively, or 12-bit quantization for the OLI sensor onboard Landsat $8^{6}$. Pre-processing includes converting calibrated DNs to absolute units of atsensor spectral radiance, top-of-atmosphere (TOA) reflectance and at-sensor brightness temperature ${ }^{6}$.

\section{Quantifying vegetation}

The most widely used spectral index for vegetation quantification is the Normalised Difference Vegetation Index, or NDVI ${ }^{7}$. Leaf chlorophyll absorbs visible light $(0.4-0.7 \mu \mathrm{m})$ for use in photosynthesis, while the leaf structure strongly reflects near-infrared light $(0.7-1.1 \mu \mathrm{m})$. Dense vegetation has an NDVI value approaching 1, while bare earth and impervious surfaces have a value around 0 . Negative values typically distinguish water bodies, which reflect visible light and absorb near-infrared.

$$
\frac{N I R-R E D}{N I R+R E D}
$$

When calculating NDVI and other vegetation indices it is important to correct for the variations that atmospheric effects have on the bands used in the calculation of NDVI, especially when comparing multiple images across time or space so that background effects from solar and atmospheric variations are minimised ${ }^{8}$. While pre-processing and atmospheric correction can remedy this, these steps are often not considered, or researchers are faced with a literature containing inconsistent terminology or outdated methods ${ }^{8}$. A further issue relates to longterm studies spanning multiple Landsat sensors. Band wavelengths vary sensor-by-sensor and therefore limit the comparison of data acquired from different sensors. This can be accounted for by the application of transformation functions to normalise the results between sensors ${ }^{9}$, however, knowing to address this requires a level of remote sensing expertise.

While Google Earth Engine will improve access to satellite data, especially for non-experts, a knowledge gap still exists where non-experts can access imagery that has not been optimally processed for a time series. This paper aims to address this issue. It presents a time-series of median annual Australia-wide NDVI data summarised for statistical areas in common use. It uses data that is both atmospherically corrected and has been normalised between sensors. The associated script also enables non-experts to access pre-processed imagery and calculate multiple spectral indices at any time-period and geographic scale more easily.

\section{Methods}

The accompanying data set contains mean annual NDVI values at the Statistical Area 1 (SA1) ${ }^{10}$ and Statistical Area 2 (SA2) ${ }^{11}$ level for the period 2001 to 2019 covering the Australian continent created from imagery processed using the accompanying script for Google Earth Engine. The raw Landsat data has been processed to produce cloud-free and atmospherically corrected satellite imagery and derived NDVI values for Landsat-5, Landsat-7 and Landsat-8 every year from 2001-2019 using the median pixel compositing method. By taking the median pixel value across all bands across the specified time period, this approach aids in the removal of clouds (which have a high value) and shadows (which have a low value). By normalising the data through compositing, imagery is created which is phrenologically and radiometrically consistent, and aids in the reduction of noise and data gaps.

Through Google Earth Engine, Landsat Tier-1 products, considered the highest available quality and suitable for time-series processing analysis, are delivered as DNs. This data can 
then either be accessed as at sensor radiance top-of-atmosphere imagery or surface reflectance imagery.

Pre-calibrated top-of-atmosphere at sensor radiance and atmospherically corrected surface reflectance imagery are available for each satellite platform through Google Earth Engine. At sensor radiance accounts for solar influences on pixel values by incorporating exoatmospheric solar irradiance, Earth-Sun distance and solar elevation angle which vary with date, time and latitude. Calibrating imagery from the original radiance values to at-sensor radiance requires a linear transform using coefficients provided in the image metadata ${ }^{6}$. To compensate for the effects that atmospheric variations have on satellite imagery, the USGS uses the LEDAPS software ${ }^{4}$ to convert the raw imagery to calibrated surface reflectance. As inputs the process requires a variety of inputs including the outputs of atmospheric simulations, a digital elevation model (DEM), total column ozone, modelled surface pressure, temperature and water vapour ${ }^{12}$. Surface reflectance imagery, through accommodating for atmospheric effects, improves comparison between multiple scenes and more accurately represents the appearance at the Earth's surface.

Table 1. Landsat mission data availability and resolution.

\begin{tabular}{l|lll}
\hline & LANDSAT 5 & LANDSAT 7 & LANDSAT 8 \\
\hline LAUNCHED & $1984-03-01$ & $1999-04-15$ & $2013-02-11$ \\
RETURNED & $2012-05-05$ & Active & Active \\
TEMPORAL RESOLUTION & 16 days & 16 days & 16 days \\
SPATIAL RESOLUTION & 30 metres & 30 metres & 30 metres \\
RADIOMETRIC RESOLUTION & 8-bit & 8-bit & 12 -bit \\
\hline
\end{tabular}

The sensors onboard the Landsat missions have evolved over time(

Table 1). Landsat 1-3 missions were equipped with the Multispectral Scanner (MSS), which recorded data in four spectral bands; two visible and two near-infrared (NIR). This was succeeded by the Thematic Mapper (TM) sensor on Landsat 4 and 5, then the Enhanced Thematic Mapper (ETM+) on Landsat 7, which provided finer spatial resolution imagery and increased radiometric resolution over the MSS sensor. These sensors also improved the spectral coverage, adding bands in the short-wave infrared (SWIR) and thermal infrared wavelengths (TIR). The latest Landsat sensor, on board Landsat 8 and the upcoming Landsat 9 platform, are equipped with the Operational Land Imager (OLI) sensor and Thermal Infrared (TIRS) sensors. The OLI augments the spectral resolution of the TM sensor with the addition of a deep blue and cirrus band, and the addition of a second thermal infrared band on the TIRS sensor. On average, the OLI reflectance is greater than the ETM+ reflectance for all bands, with the greatest differences in the NIR and SWIR bands ${ }^{9}$. Although differences between each generation of Landsat sensors are quite small, they may have a significant impact on results depending on the application. Statistical functions to transform between each sensor bands and derived NDVI values published by Roy et al ${ }^{9}$ are included for the NDVI data set to improve temporal continuity (

Processed NDVI imagery covering each Australian state and territory was exported to QGIS where statistics were calculated for each ASGS SA1 and SA2 boundary: the smallest geographic units in Australia for the release of census data.

Table 2). 
Processed NDVI imagery covering each Australian state and territory was exported to QGIS where statistics were calculated for each ASGS SA1 and SA2 boundary: the smallest geographic units in Australia for the release of census data.

Table 2. Landsat sensor transformation functions to improve inter-sensor continuity for the Normalised Difference Vegetation Index.

\begin{tabular}{l|ll}
\hline IMAGE PROCESSING & PLATFORM & NDVI TRANSFORMATION FUNCTIONS \\
\hline TOP-OF-ATMOSPHERE & LANDSAT-5 & OLI $=0.0306+0.9824$ ETM \\
& & \\
& LANDSAT-7 & OLI $=0.0490+0.9352$ ETM+ \\
& LANDSAT-8 & ETM $+=-0.0110+0.9690$ OLI \\
\hline SURFACE REFLECTANCE & LANDSAT-5 & OLI $=0.0149+1.0035 \mathrm{ETM}$ \\
& LANDSAT-7 & OLI $=00235+09723$ ETM + \\
& LANDSAT-8 & ETM $+=0.0029+0.9589$ OLI \\
\hline
\end{tabular}

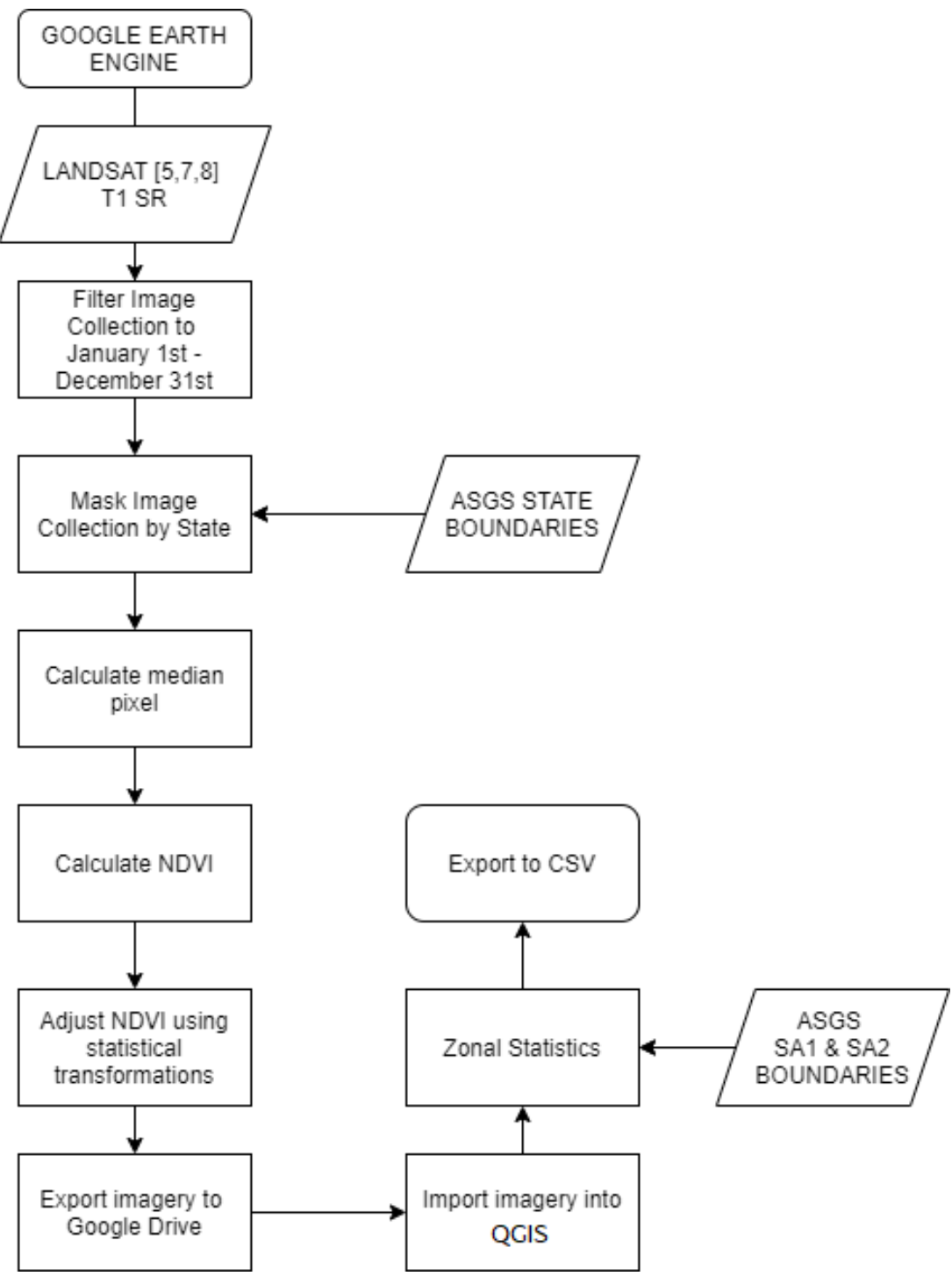

Figure 1. Landsat data processing workflow in Google Earth Engine and QGIS. 
This workflow (Figure 1) was repeated every year for each Australian state for the years 20012019 to produce a collection of spreadsheets containing statistical measures of greenness (Table 3) including the mean NDVI value across each statistical area, the standard deviation, the minimum value and maximum. The resulting tables were exported to CSV format.

Table 3. Statistical measures for median annual NDVI values calculated in QGIS for each Statistical Area.

\begin{tabular}{l|l}
\hline COLUMN & VALUE \\
\hline SA1code/SA2code & Statistical Area 1 or 2 code \\
countNDVI & Count of NDVI values in Statistical Area \\
meanNDVI & Mean NDVI value in Statistical Area \\
medianndvi & Median NDVI value in Statistical Area \\
sdNDVI & Standard Deviation of NDVI values in Statistical Area \\
minNDVI & Minimum NDVI value in Statistical Area \\
maxNDVI & Maximum NDVI value in Statistical Area \\
\hline
\end{tabular}

For the years 2001-2013, either Landsat-5 or Landsat-7 were selected based on which sensor showed the best image coverage. The Scan Line Corrector (SLC) on board Landsat-7 failed on May 31, 2003. As a result, Landsat-7 imagery after this date may contain data gaps and striping, therefore should both platforms have complete image coverage for the state, Landsat 5 would be selected in preference to Landsat 7. For the years 2014-2019, Landsat-8 was consistently chosen. Some statistical areas contain data gaps, primarily caused by consistent cloud cover across the year. In these instances, the Statistical Areas in the resulting data will contain null values.

\section{Data Records}

Two datasets describing annual median NDVI summary statistics from 2001-2019 are available in .csv format from the Open Science Foundation (https://osf.io/5h3dg). The first summarises positive NDVI for 2011 SA1s. The second summarises positive NDVI for 2011 SA2s. Data was stratified by State using the Australian Statistical Geography Standard (ASGS) 2011 dataset $^{13}$. The summary statistics calculated in QGIS for each Statistical Area include count, mean, median, standard deviation, minimum and maximum. Each data record also includes either an SA1 or SA2 code, enabling linkage to geographic boundaries available for download from the Australian Bureau of Statistics.

\section{Technical Validation}

With each new generation of Landsat satellites come sensor and calibration improvements, including adjustments to the spectral resolution which need to be accounted for when conducting long term time series studies across multiple sensors. Statistical functions to transform between each sensor bands and derived NDVI values published by Roy et al ${ }^{9}$ are included for the NDVI data set to improve temporal continuity.

To validate the consistency of imagery between sensors before and after adjustment, threeyear median composites were created covering the state of Victoria, Australia, across two time periods. First, from 2007-2009 when both Landsat-5 and Landsat-7 are active (Figure 2), and second, from 2017-2019 when both Landsat-7 and Landsat-8 are active (Figure 3). Results were stratified by SA1 boundaries and the mean NDVI value for each SA1 is compared. 
2007-2009 comparison of adjusted NDVI values for Landsat-5 and Landsat-7 Surface Reflectance Imagery

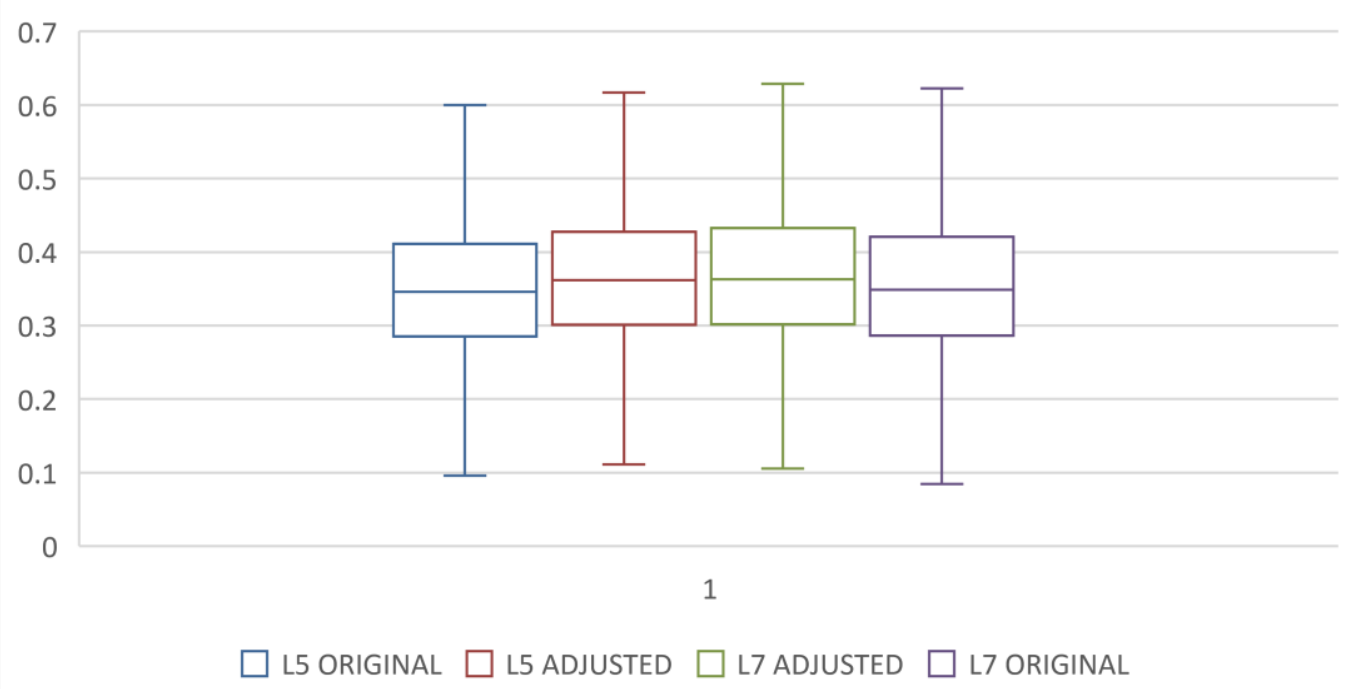

Figure 2. Comparison of NDVI values for Victoria, Australia calculated from Landsat-5 and Landsat-7 composite surface reflectance imagery from three consecutive years, 2007-2009. Adjusted values for each platform are shown in the centre.

Table 4. Quantile distribution of NDVI values for Landsat-5 and Landsat-7 for the period 2007-2009 across Victoria, Australia. showing original values and after applying statistical adjustments published by Roy et al. ${ }^{9}$

\begin{tabular}{l|lllll}
\hline PLATFORM & Q1 & Q2 & MEDIAN & Q4 & Q5 \\
\hline LANDSAT-5 & 0.0956 & 0.2850 & 0.3458 & 0.4101 & 0.6001 \\
LANDSAT-7 & 0.0846 & 0.2863 & 0.3488 & 0.4201 & 0.6226 \\
LANDSAT-5 & 0.1112 & 0.3009 & 0.3618 & 0.4273 & 0.6171 \\
(ADJUSTED) & & & & & \\
LANDSAT-7 & 0.1057 & 0.3018 & 0.3627 & 0.4323 & 0.6289 \\
(ADJUSTED) & & & & & \\
\hline
\end{tabular}




\section{7-2019 comparison of adjusted NDVI values for Landsat-7 and Landsat-8 Surface Reflectance Imagery}

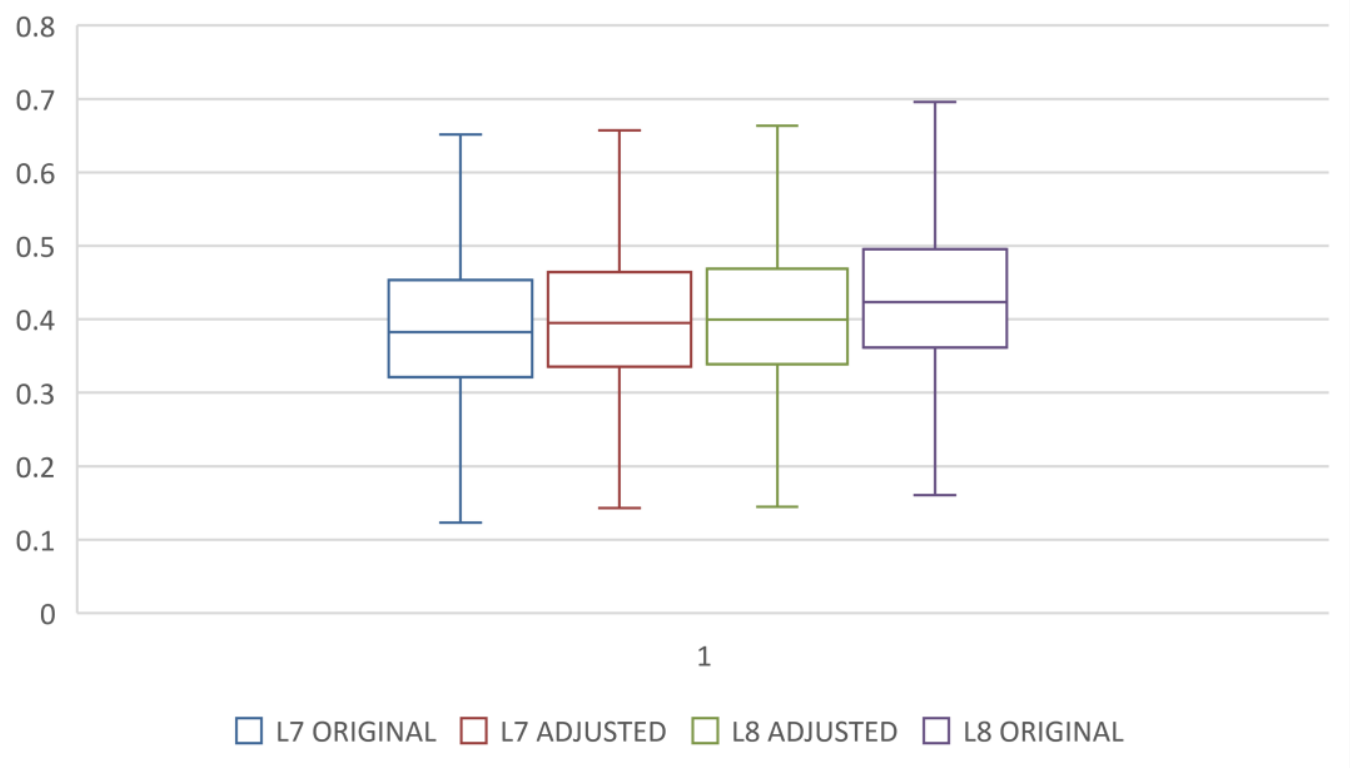

Figure 3. Comparison of NDVI values for Victoria, Australia calculated from Landsat-7 and Landsat-8 composite surface reflectance imagery from three consecutive years, 2017-2019. Adjusted values for each platform are shown in the centre.

Table 5. Quantile distribution of NDVI values for Landsat-7 and Landsat-8 for the period 2017-2019 across Victoria, Australia. showing original values and after applying statistical adjustments published by Roy et al. ${ }^{9}$

\begin{tabular}{l|lllll}
\hline PLATFORM & Q1 & Q2 & MEDIAN & Q4 & Q5 \\
\hline LANDSAT-7 & 0.1230 & 0.3211 & 0.3823 & 0.4534 & 0.6515 \\
LANDSAT-8 & 0.1606 & 0.3613 & 0.4238 & 0.4954 & 0.6958 \\
LANDSAT-7 & 0.1431 & 0.3357 & 0.3952 & 0.4643 & 0.6570 \\
$\begin{array}{l}\text { (ADJUSTED) } \\
\text { LANDSAT-8 }\end{array}$ & 0.1446 & 0.3391 & 0.3994 & 0.4690 & 0.6633 \\
(ADJUSTED) & & & & & \\
\hline
\end{tabular}

The statistical adjustments published by Roy et al ${ }^{9}$ increase the raw NDVI values for Landsat-5 and Landsat-7 (Table 4), while lowering the raw values for Landsat-8 (Table 5). The adjustments had the largest effect on Landsat-8, significantly reducing the percentage difference when compared to Landsat-7 (Table 6). The reduced difference between adjusted values and the raw imagery indicates that continuity between sensors has been improved.

Table 6. Percentage difference between quantile distribution of NDVI values for Landsat-7 and Landsat-8 for the period 2017-2019 across Victoria, Australia. showing original values and after applying statistical adjustments published by Roy et al. ${ }^{9}$

\begin{tabular}{|c|c|c|c|c|c|}
\hline PERCENTAGE DIFFERENCE & Q1 & Q2 & MEDIAN & Q4 & Q5 \\
\hline LANDSAT-5 \& LANDSAT-7 & $-12.21 \%$ & $0.46 \%$ & $0.86 \%$ & $2.41 \%$ & $3.68 \%$ \\
\hline LANDSAT-7 \& LANDSAT-8 & $26.52 \%$ & $11.78 \%$ & $10.30 \%$ & $8.85 \%$ & $6.58 \%$ \\
\hline $\begin{array}{lll}\text { LANDSAT-5 } & \& & \text { LANDSAT-7 } \\
\text { (ADJUSTED) } & & \end{array}$ & $-5.07 \%$ & $0.30 \%$ & $0.25 \%$ & $1.16 \%$ & $1.89 \%$ \\
\hline $\begin{array}{l}\text { LANDSAT-7 } \quad \& \quad \text { LANDSAT }-8 \\
\text { (ADJUSTED) }\end{array}$ & $1.04 \%$ & $1.01 \%$ & $1.06 \%$ & $1.01 \%$ & $0.95 \%$ \\
\hline
\end{tabular}




\section{Usage Notes}

This dataset of NDVI values for Australian statistical areas is intended to provide researchers with pre-processed data for vegetation studies across multiple years between 2001 and 2019. The accompanying script for Google Earth Engine simplifies the process of accessing analysis ready medium resolution imagery for longitudinal ecological or urban greenspace studies. Imagery available for export includes composited Landsat or Sentinel-2 imagery and derived spectral indices commonly used in vegetation or urban studies.

\section{Code Availability}

The script for Google Earth Engine used to create this dataset is available on GitHub (https://github.com/simondramsey/GEE-Image Export/tree/GEE Image Export V1).

\section{Acknowledgements}

SM was supported by Australian National Health and Medical Research Council fellowship (\#1121035) and a University of Melbourne Faculty of Medicine, Dentistry and Health Sciences Research Fellowship. This project was supported by funds from the Centre for Health Equity, Melbourne School of Population and Global Health, University of Melbourne.

\section{Author contributions}

SR contributed to project development, wrote the scripts, analysed the data, and drafted the manuscript.

SM conceived the project, secured funding, analysed the data, and contributed to the manuscript.

\section{Competing interests}

The authors declare no competing interests.

\section{References}

1. Gorelick, N. et al. Google Earth Engine: Planetary-scale geospatial analysis for everyone. Remote Sensing of Environment 202, 18-27 (2017).

2. Roy, D. P. et al. Landsat-8: Science and product vision for terrestrial global change research. Remote Sensing of Environment 145, 154-172 (2014).

3. Miller, H. M. et al. The users, uses, and value of Landsat and other moderateresolution satellite imagery in the United States-Executive report: U.S. Geological Survey Open-File Report 2011-1031. 42 (2011).

4. Schmidt, G. Landsat Ecosystem Disturbance Adaptive Processing System (LEDAPS) Algorithm Description. (2013).

5. Vermote, E., Roger, J. C., Franch, B. \& Skakun, S. LaSRC (Land Surface Reflectance Code): Overview, application and validation using MODIS, VIIRS, LANDSAT and Sentinel 2 data's. in IGARSS 2018 - 2018 IEEE International Geoscience and Remote Sensing Symposium 8173-8176 (IEEE, 2018). doi:10.1109/IGARSS.2018.8517622.

6. Chander, G., Markham, B. L. \& Helder, D. L. Summary of current radiometric calibration coefficients for Landsat MSS, TM, ETM+, and EO-1 ALI sensors. Remote Sensing of Environment 113, 893-903 (2009).

7. Rouse, W. \& Haas, R. H. Monitoring Vegetation Systems in the Great Plains using ERTS. In Third ERTS Symposium; NASA: Washington, DC, USA (1973). 
8. Young, N. E. et al. A survival guide to Landsat preprocessing. Ecology 98, 920-932 (2017).

9. Roy, D. P. et al. Characterization of Landsat-7 to Landsat-8 reflective wavelength and normalized difference vegetation index continuity. Remote Sensing of Environment 185, 5770 (2016).

10. Australian Bureau Of Statistics. Statistical Area Level 1 (SA1) ASGS Ed 2011 Digital Boundaries in ESRI Shapefile Format. (2016).

11. Australian Bureau Of Statistics. Statistical Area Level 2 (SA2) ASGS Ed 2011 Digital Boundaires in ESRI Shapefile Format. (2016).

12. Kalnay, E. et al. The NCEP/NCAR 40-Year Reanalysis Project. Bulletin of the American Meteorological Society 77, 437-472 (1996).

13. Australian Bureau Of Statistics. State (S/T) ASGS Ed 2011 Digital Boundaries in ESRI Shapefile Format. (2016). 